\title{
ASELLUS AQUATICUS L. EN LA PENÍNSULA IBÉRICA
}

\author{
Carmen Zamora Muñoz y Javier Alba-Tercedor \\ Departamento de Biología Animal y Ecología, Facultad de Ciencias, Universidad de Granada, 18071 - Granada (España)
}

Palabras clave: Isopoda, Asellus aquaticus, selección de habitat, distribución, Península Ibérica.

\section{ABSTRACT}

\section{ASELLUS AQUATICUS L. IN THE IBERIAN PENINSULA}

The Isopod Asellus aquaticus is a common species in Europe, but up to now it had not been recorded in the Iberian Peninsula.

The ranges inhabited by A. aquaticus in the study area were delimited by studying its distribution in the Genil basin, and by measuring the physico-chemical factors in the sampling sites.

As recorded in other areas, the presence of A. aquaticus in the Genil river basin is associated with downstream stretches of river, with low altitude, high flow and organic pollution of domestic and agriculturalorigin.

\section{RESUMEN}

Se amplía el área de distribución del Isópodo Asellus aquaticus, especie comun en Europa pero que hasta el momento no había sido citada en la Península Ibérica.

A partir del estudio de la distribución de esta especie en la cuenca del río Genil y de los factores fisico-químicos medidos en las estaciones de muestreo, se delimitaron las características del hábitat de A. aquaticus en el área de estudio.

Coincidiendo con lo observado por otros autores, la presencia de A. aquaticus en la cuenca del río Genil se halla ligada a tramos de ríos alejados de la cabecera, de escasa altitud, elevado caudal y con contaminación orgánica considerable procedente de la actividad doméstica y de la agricultura.

\section{INTRODUCCIÓN}

En la Península Ibérica existe una gran diversificación de Asélidos de agua dulce, con un elevado porcentaje de endemismos y de especies emparentadas con la fauna circummediterránea. Sin embargo, hasta el presente trabajo no se habían capturado especies de origen nórdico (ver G. DE BIKUÑA, 1989; HENRY \& MAGNIEZ, 1978).

Durante el estudio de la fauna macroinvertebrada de la cuenca hidrográfica del Alto Genil (prov. de Granada) realizado en el período comprendido entre Marzo de 1988 y Febrero de 1990 se capturaron Isópodos pertenecientes a la familia Asellidae. De acuerdo con los trabajos de ARGANO (1979) y BIRSTEIN (1964), y tras el estudio de los pleópodos I y II de los machos y del II de las hembras, observamos que se trataba de Asellus aquaticus (LINNAEUS, 1758) sensu RACOVITZA 1919, especie difundida por toda Europa y no citada hasta ahora en la Península Ibérica (ARGANO, 1979; BIRSTEIN, 1964;
G. DE BIKUÑA, 1989; G. DE BIKUÑA \& ASENSIO, 1991; HENRY \& MAGNIEZ, 1978; MARGALEF, 1983).

En la misma cuenca se capturó de forma puntual y aislada Proasellus meridianus (RACOVITZA, 1919), en localidades donde nunca se encontró A. aquaticus.

Con objeto de ampliar el conocimiento que se tiene de $\boldsymbol{A}$. aquaticus se ha realizado un estudio de las características fisico-químicas que determinan su distribución en los cursos de agua en que se ha capturado esta especie, así como de la fauna asociada con ella.

\section{ÁREA DE ESTUDIO}

El presente estudio se realizó en la cuenca hidrográfica del Alto Genil, considerándose como tal la cuenca vertiente al río Genil hasta el embalse de Iznájar. Dicha cuenca, que forma parte de la cuenca del Guadalquivir, ocupa una superficie de $4.500 \mathrm{Km}^{2}$, aproximadamente, localizándose la mayor parte de ella dentro de la provincia de Granada (Fig. 1). 
Dado el carácter montañoso del área de estudio son numerosos los tributarios que recibe el cauce principal, el río Genil (Fig. 1). Dichos afluentes presentan regímenes hidrológicos diversos y discurren por materiales muy diferentes. Los ríos nacidos en Sierra Nevada (sur-este de la cuenca) tienen regímenes nivales o pluvionivales y drenan en sus cabeceras por micaesquistos mayoritariamente. Las cuencas medias de estos ríos así como las cabeceras de los ríos de la zona sur, noroeste y sureste recorren principalmente materiales carbonatados (dolomías y calizodolomías), estando su caudal directamente relacionado con el régimen de lluvias, aunque en alguno de ellos las aportaciones subterráneas son mayoritarias. Por último, los valles de los cursos más deprimidos topográficamente (Genil, Cubillas, Colomera, Velillos, Cacín y Alhama, entre otros) están formados por materiales detríticos (UNIV. GRANADA, 1990; ZAMORA-MUNOZ, 1992).

La mayor parte de los ríos estudiados están contaminados tanto por vertidos urbanos, como agrícolas o industriales (industrias agroalimentarias, sobre todo), si bien, y dado el escaso desarrollo industrial de la zona, la principal causa de degradación son los vertidos de aguas residuales urbanas sin depurar a los cauces (S.A.S.-UNIV. GRANADA, 1991; ZAMORA-MUÑOZ, 1992).

\section{MATERIAL Y MÉTODOS}

Entre el curso principal del río Genil y 26 tributarios de la cuenca se establecieron un total de 60 estaciones, que se muestrearon estacionalmente entre Marzo de 1988 y Febrero de 1990.

La recogida de macroinvertebrados se realizó de forma cualitativa mediante muestreo tipo "kick" con redes de 0,5 y 0,3 mm de abertura de malla. En cada una de las estaciones se muestreaban todos los microhábitats existentes en el cauce y el contenido de las redes iba siendo depositado periódicamente en bateas, para evitar la pérdida de organismos con su colmatación. Cada muestreo se consideraba terminado cuando, tras sucesivas redadas, las capturas no aportaban nuevos taxones.

A la vez que se recogía el material biológico se midieron el $\mathrm{pH}$, el caudal y la temperatura. Asimismo se tomaron muestras de agua para el análisis en el laboratorio de los siguientes parámetros: conductividad, sulfatos, nitritos, nitratos, amonio, fosfatos, calcio, magnesio, cloruros, sodio, potasio, hierro (111), cobre (II), cinc (11), plomo (II), níquel (11), suma de plaguicidas, detergentes y aceites y grasas (las técnicas de análisis utilizadas se detallan en UNIV. GRANADA, 1990).

La calidad biológica del agua fue calculada mediante el índice BMWP' (ALBA-TERCEDOR \& SANCHEZ-ORTE-

FIGURA 1. Situación de la cuenca hidrográfica del Alto Genil dentro de la cuenca del Guadalquivir (con trazo mas oscuro se señala el río Genil), estaciones de muestreo (puntos negros) y localidades en que se capturaron Asellus aquaticus $(+)$ y Proasellus meridianus $(\leftarrow)$.

FIGURE 1. Location of the hydrographic basin of the Alto Genil with in the Guadalquivir basin (course of the Genil river with dark outline), sampling sites (black points) and localities where Asellus aquaticus (+) and Proasellus meridianus (*) were caught.
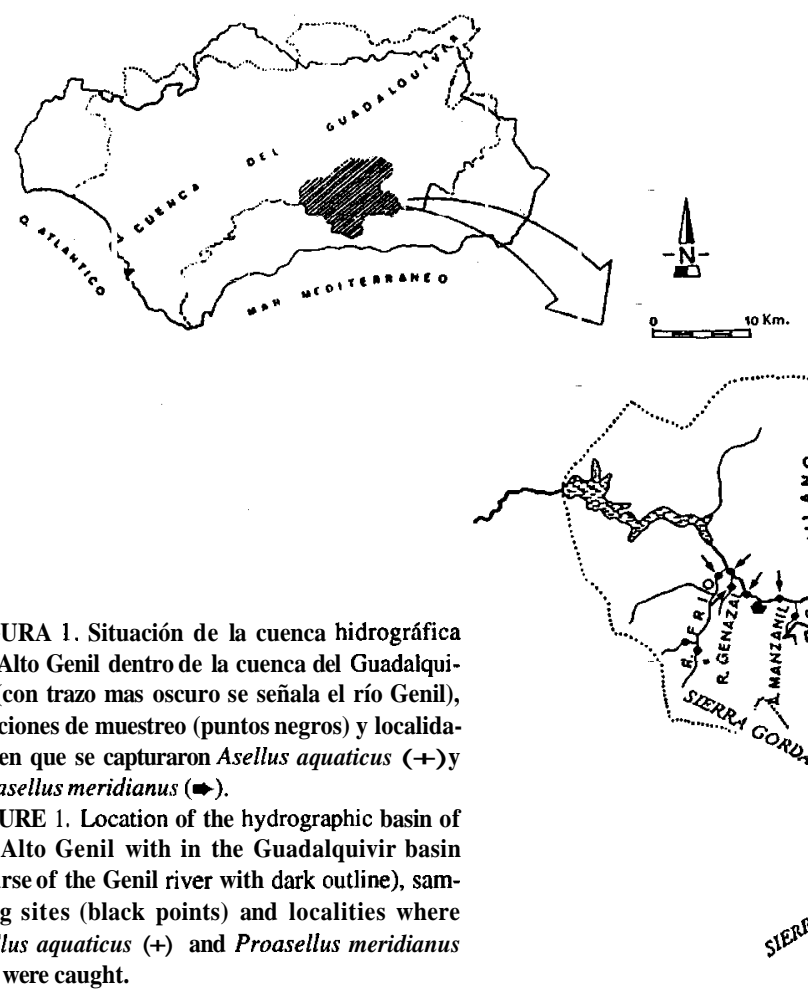
GA, 1988) al que se le incluyeron nuevas familias que no se encontraban en el trabajo original (ZAMORA-MUÑOZ, 1992).

Para conocer qué variables fisico-químicas explican la presencia/ausencia de A. aquaticus en el área de estudio se utilizó un análisis discriminante múltiple (MDA) (LUDWIG \& REYNOLDS, 1988). Se utilizó el método por pasos por medio del cual es posible aislar, de entre todas las variables, las que significativamente discriminan entre la presencia o ausencia de la especie. Este análisis proporciona ecuaciones para predecir, en base a las características fisico-químicas, la probabilidad de que en una estación de muestreo se encuentre o no dicha especie. Previamente a la realización del análisis se normalizaron aquellas variables que lo necesitaron. La lista de variables utilizadas en el análisis así como las transformaciones adecuadas se encuentran en la Tabla 1.

TABLA 1. Variables fisico-químicas utilizadas en el análisis discriminante múltiple y las transformaciones para su aproximacióna una normal.

TABLE 1. Physico-chenical variables in the multiple discriminant analysis and transformation used to aproximate them to a normal function.

\begin{tabular}{ll}
\hline VARIABLE & \multicolumn{1}{c}{ TIPO DE } \\
FISICO-QUÍMICA & TRANSFORMACIÓN \\
\hline Altitud & $-1 / 6 \sqrt{x}$ \\
Distanciaal origen & $6 \sqrt{x}$ \\
Pendiente & $-1 / 6 \sqrt{x}$ \\
Caudal & $\log (x+1)$ \\
Temperatura & $x$ \\
pH & $3 \sqrt{x}$ \\
Conductividad & $\log (x+1)$ \\
Sulfatos & $6 \sqrt{x}$ \\
Nitritos & $-1 /^{10} \sqrt{x}$ \\
Nitratos & $3 \sqrt{x}$ \\
Amonio & $-1 / 5 \sqrt{x}$ \\
Fosfatos & $-1 /^{10} \sqrt{x}$ \\
Calcio & $6 \sqrt{x} *$ \\
Magnesio & $3 \sqrt{x}$ \\
Cloruros & $-1 /^{10} \sqrt{x}$ \\
Sodio & $\log (x+1)$ \\
Potasio & $-1 /^{10} \sqrt{x}$ \\
&
\end{tabular}

\section{RESULTADOS}

De un total de 60 estaciones de muestreo establecidas en la cuenca del Genil A. aquaticus se capturó en 14 de ellas. Estas estaciones presentaron rangos de altitud y pendiente bastante estrechos (440-550 m; 0,01-0,05\%), correspondlentes a zonas alejadas de las cabeceras (Distancia al origen media $=63 \mathrm{~km}$ ) y de caudal considerable (media $=3.847 \mathrm{l} / \mathrm{s}$; Tabla 2 ). La mayoría pertenecen al eje principal del río Genil aguas abajo de la ciudad de Granada y el resto a tramos próximos a la desembocadura de algunos de sus afluentes (Apéndice 1; Fig. 1). Todas ellas se caracterizaron por presentar substrato pedregoso con abundante depósito de lodo.

El rango de temperatura en que se encontró la especie fue muy amplio (Tabla 2), como consecuencia de que se capturaron ejemplares en todas las estaciones del año. Sin embargo, la media correspondió a una temperatura cálida (media $=16,1 \mathrm{C}$ ).

Con respecto a la mineralización de las aguas, A. aquaticus toleró un rango muy amplio de concentración, tanto de cationes como de aniones, aunque los valores medios se encontraron dentro de los términos de aguas bien mineralizadas (ver valores de conductividad, calcio y sulfatos en Tabla 2).

A. aquaticus ha sido encontrado en estaclones sometidas a contaminación, habiéndose capturado ejemplares dentro de valores muy extremos. Aunque en las aguas donde habitó esta especie se detectaron niveles anómalos de metales pesados, pesticidas y grasas, la principal fuente contaminante fueron los vertidos urbanos (ver Area de estudio), como se observa en los valores obtenidos para los parámetros indicadores de materia orgánica (compuestos nitrogenados y fosfatos) y para los que esta especie mostró gran tolerancia (ver valores medios en Tabla 2).

Asimismo, con respecto a los valores del índice biológico BMWP' obtenidos, encontramos a A. aquaticus en un rango amplio de calidad de las aguas, desde ligeramente a muy contaminadas, aunque con preferencia por las aguas contaminadas de calidad "dudosa" (media= 38). Es de destacar que nunca se capturaron ejemplares en aguas fuertemente contaminadas (en situación "muy crítica").

Para averiguar cuales de los parámetros fisico-químicos, de entre los que consideramos más relevantes (detallados en la Tabla 1), explican mejor la presencia/ausencia de A. aquaticus en la zona de estudio, realizamos un análisis discriminante múltiple (MDA). Obtuvimos una función discriminante (o canónica) estadísticamente significativa para $\mathbf{p}<0,001$ (Tabla $3 \mathrm{~A}$ ), que explica el $100 \%$ de la varianza total. De todos los parámetros fisico-químicos utilizados en el análisis, cinco fueron los que mejor explicaron la presencia o ausencia de $\boldsymbol{A}$. aquaticus: distancia al origen, altitud, caudal, fosfatos y amonio, siendo sobre todo las cuatro primeras variables las que tienen mayor importancia, al tener coeficientes canónicos mayores (Tabla 3B). Es de destacar que los grupos presencia/ausencia de esta especie resultaron significativamente distintos sólo teniendo en cuenta estas variables $(\mathrm{F}=31,32 ;$ g.l. $=5,143$; $\mathrm{p}<0,001$ ).

De forma general, conviviendo con A. aquaticus se encontraron varias especies de Moluscos (Potamopyrgus jenkinsi, 
TABLA 2. Número de casos (N), valores mínimos, máximos, media y error estándar (E.S.) de las variables fisico-químicas y del índice biológico BMWP' en los que se capturó Asellus aquaticus.

TABLE 2. Number of cases (N), minimum, maximum values, mean and standard error (E.S.) of physico-chemical variables and blologlcal index BMWP' where Asellus aquaticus was caught.

\begin{tabular}{|c|c|c|c|c|c|}
\hline Variables físico-químicas & $\mathrm{N}$ & Min. & Max & Media & E. S. \\
\hline Altitud (m) & 69 & 440,00 & 550,00 & 500,25 & 3,95 \\
\hline Distancia al origen $(\mathrm{km})$ & 79 & 2,30 & 106,20 & 63,26 & 3.46 \\
\hline Pendiente $(\%)$ & 79 & 0,00 & 0,05 & 0,03 & 0,00 \\
\hline Caudal $(1 / s)$ & 79 & 18,00 & 13172,00 & 3847,60 & 360.97 \\
\hline Temperatura $\left({ }^{\circ} \mathrm{C}\right)$ & 79 & 9,00 & 25,00 & 16,09 & 0,45 \\
\hline $\mathrm{pH}$ & 62 & 7,30 & 8,60 & 7,82 & 0,04 \\
\hline Conductividad $\left(\mu \mathrm{mhos} / \mathrm{cm} 20^{\circ} \mathrm{C}\right)$ & 68 & 585,00 & 3650,00 & 1478,98 & 64.85 \\
\hline Sulfatos (mg/l) & 79 & 16,00 & 1253,00 & 347,31 & 26.15 \\
\hline Nitritos (mg/1) & 60 & 0,00 & 3,92 & 0,88 & 0,13 \\
\hline Nitratos $(\mathrm{mg} / \mathrm{l})$ & 79 & 0,00 & 224,00 & 43,65 & 4.83 \\
\hline Amonio (mg/l) & 79 & 0,00 & 43.90 & 1,98 & 0.59 \\
\hline Fosfatos (mg/l) & 79 & 0,00 & 3.66 & 0,52 & 0,08 \\
\hline Calcio (mg/l) & 60 & 45,00 & 562,00 & 148,82 & 7,34 \\
\hline Magnesio (mg/l) & 79 & 0,00 & 248,00 & 59,41 & 3,66 \\
\hline Cloruros (mg/l) & 79 & 18.00 & 636,00 & 143,48 & 10,21 \\
\hline Sodio (mg/l) & 79 & 4,00 & 670,00 & 98,60 & 13.29 \\
\hline Potasio (mg/l) & 79 & 1,00 & 12.00 & 4.04 & 0,20 \\
\hline Нiеғғо $(\mu g / l)$ & 79 & 0,00 & 1650,00 & 201,24 & 31,94 \\
\hline Cobre $(\mu \mathrm{g} / \mathrm{l})$ & 70 & 0,00 & 99,00 & 9,31 & 2,15 \\
\hline $\operatorname{Cinc}(\mu \mathrm{g} / \mathrm{l})$ & 70 & 0,00 & 130,00 & 28,69 & 3,14 \\
\hline Plomo (pg/l) & 70 & 0,00 & 24,00 & $2 ; 86$ & $0 ; 83$ \\
\hline Níquel $(\mu \mathrm{g} / \mathrm{l})$ & 70 & 0,00 & 31,00 & 6,37 & 0.86 \\
\hline Suma de plaguicidas $(\mu \mathrm{g} / \mathrm{l})$ & 70 & 0,00 & 1.95 & 0.13 & 0,04 \\
\hline Detergentes $(\mathbf{m g} / \mathbf{l})$ & 46 & 0,00 & 5,82 & 0,40 & 0,11 \\
\hline Aceites y grasas $(\mathrm{mg} / \mathrm{l})$ & 70 & 0,00 & 216.50 & 12,20 & 3,93 \\
\hline Indice BMWP' & 79 & 9,00 & 96.00 & 37,81 & 2.16 \\
\hline
\end{tabular}

TABLA 3. Resultados del análisis discriminante múltlple (MDA) por pasos entre los grupos presencia/ausencia de Asellus aquaticus y 17 variables fisico-químicas. A: valores estadísticos de la función discriminante obtenida. B: coeficientes estandarizados de la función discriminante (desde el origen de coordenadas) para las variables fisico-químicasque explican dicha función.

TABLE 3. Stepwise multiple discriminant analysis (MDA) results between Asellus aquaticus presence/absence groups and 17 physicochemical variables. A: statistical values

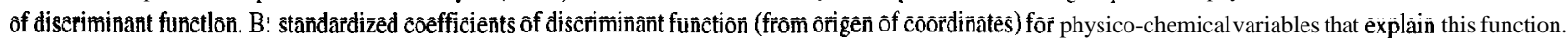

A:

\begin{tabular}{lcccccc}
\hline $\begin{array}{l}\text { Func. } \\
\text { Discriminante }\end{array}$ & $\begin{array}{c}\text { Eigen- } \\
\text { value }\end{array}$ & $\begin{array}{c}\mathrm{R} \\
\text { Canonica }\end{array}$ & $\begin{array}{c}\text { Wilks' } \\
\text { Lambda }\end{array}$ & Chi-Sqr. & g.l. & $\mathrm{P}$ \\
\hline 1 & $\mathbf{1 , 1 1}$ & 0,73 & 0,47 & 108.53 & 5 & 0,00
\end{tabular}

B:

Coeficientes de la

Variables función discriminante

\begin{tabular}{lc}
\hline Distancia al origen & 0,5367 \\
Altitud & -0.4211 \\
Caudal & 0,5898 \\
Fosfatos & $-0,5574$ \\
Amonio & 0,2923
\end{tabular}


Physella acuta y Ancylus fluviatilis), de Anélidos (Eiseniella tetrahedra, Tubificidae, Naididae, Helobdella stagnalis y Erpobdellidae), de Dípteros (Chironomus gr. thummi y Simuliidae, sobre todo), de Efemerópteros (Baetis pavidus, B. rhodani y Caenis luctuosa), una especie de Anfípodos (Echinogammarus obtusidens) y una de Tricópteros (Hydropsyche exocellata).

\section{DISCUSIÓN}

En nuestra zona de estudio, Asellus aquaticus vive dentro de un amplio rango de valores aunque, por regla general, lo encontramos en localidades donde las características fisicoquímicas indicaron una clara alteración de las aguas.

La alta tolerancia de esta especie a contaminaciones orgánicas es ampliamente conocida y por ello es utilizada como indicadora en la sucesión de organismos tras un fuerte vertido contaminante de naturaleza orgánica. De hecho, la presencia de $\boldsymbol{A}$. aquaticus da nombre a la zona del río en que aparece y que se encuentra en proceso de autodepuración: "zona del Asellus" (HELLAWELL, 1986; HYNES, 1960; JEFFRIES \& MILLS, 1990; MASON, 1984). En esta zona, además de esta especie, se ha descrito que es común encontrar Hirudíneos, Moluscos y Gammáridos (op. cit.), grupos asociados también con A. aquaticus en nuestra zona de estudio. Asimismo, su presencia estuvo ligada a la de otras especies dominantes en tramos bajos y eutróficos de los ríos en la Península Ibérica: Baetis pavidus, Caenis luctuosa e Hydropsyche exocellata (ALBA-TERCEDOR \& JIMENEZ-MILLAN, 1987; BASAGUREN, 1988; BASAGUREN \& ORIVE, 1990; GARCIA DE JALON, 1986; GONZALEZ DEL TANAGO \& GARCIA DE JALON, 1984; PUIG et al., 1981; ZAMORA-MUÑOZ, 1992; entre otros).

El hecho de que las variables físicas: distancia al origen, altitud y caudal, sean importantes a la hora de discriminar entre la presencia y ausencia de $\mathrm{A}$ aquaticus es debido a que esta especie es mucho más abundante en aguas remansadas donde vive entre los tallos y hojas de los macrófitos (BIRSTEIN, 1964), alimentándose tanto de materia vegetal viva como en descomposición (MALTBY, 1991). Esas condiciones se encuentran en nuestra área de estudio en la zona de hiporhithron-epipotamon del río Genil y en la desembocadura de algunos de sus afluentes, donde la escasa pendiente lleva a que estos tramos tengan una corriente casi laminar y a que se acumule la materia orgánica particulada gruesa. Estas zonas correspondieron, a su vez, con los tramos más contaminados.

La tolerancia de esta especie en nuestra zona de estudio por aguas bien mineralizadas, así como por un amplio rango de temperatura, coincide con lo observado en la literatura (BIRSTEIN, 1964).

En la zona de estudio se capturó también, aunque de forma puntual y escasa, Proasellus meridianus (Apéndice 1), especie que, con anterioridad, había sido capturada en el norte de la Península Ibérica ligada a cursos de agua de escasa altitud, elevada carga orgánica y muy alto grado de mineralización (G. DE BIKUÑA \& ASENSIO, 1991). Su presencia en la cuenca del río Genil está más relacionada con surgencias de agua y tramos de ríos no contaminados (ver ZAMORA-MUÑOZ, 1992, subnom. Proasellus coxalis), coincidiendo con lo observado por HENRY \& MAGNIEZ (1983) en Francia. Debido a la escasez de capturas no podemos afirmar, como hacen HENRY \& MAGNIEZ (op. cit.), que dicha especie sea sustituida en las zonas contaminadas por la especie invasora $\mathbf{A}$. aquaticus. Sin embargo, el hecho de que nunca se encontraran conviviendo parece estar de acuerdo con dichas observaciones.

\section{Aspectos biogeográficos}

Asellus aquaticus es una especie emparentada con los Asélidos de Siberia Oriental y Japón que desde Asia, aprovechando el deshielo postglaciar cuaternario, se extendió masivamente por Europa septentrional y media (BIRSTEIN, 1964; HENRY \& MAGNIEZ, 1978). BIRSTEIN (op. cit.) explicaba su ausencia en la Península Ibérica por la relativa reciente penetración de esta especie en Europa. Sin embargo, tras este estudio, en el que se observó una zona de colonización bastante amplia y unas poblaciones bien establecidas, creemos que, en parte, su ausencia puede haber sido debida a la falta de conocimiento que aún se tiene de la fauna dulceacuícola de nuestra Península.

\section{AGRADECIMIENTOS}

Queremos expresar nuestro agradecimiento a todos los que participaron en las campañas de muestreo del Genil, y en especial a: I. Guisasola, F. Madrid Vinuesa, J. Martín, J. Picazo, C.E. Sáinz-Cantero y A. Sánchez-Ortega. También a los miembros del Instituto Andaluz de Geología Mediterránea y del Laboratorio Laín que realizaron los análisis químicos. A J. Picazo, por la realización de los mapas. Al Ministerio de Educación y Ciencia y a la Universidad de Granada por las becas predoctoral y postdoctoral concedidas, respectivamente, por estos organismos al primero de los autores. A la Dirección General de Obras Hidráulicas del M.O.P. Upor el proyecto de investigación concedido y a partir del cual se ha subvencionado este trabajo. 


\section{BIBLIOGRAFÍA}

ALBA-TERCEDOR, J. \& F. JIMENEZ-MILLAN, 1987. Evaluación de las variaciones estacionales de la calidad de las aguas del río Guadalfeo basada en el estudio de las comunidades de macroinvertebrados acuáticos y de los factores fisico-químicos. LUCDEME III. ICONA. Monografía 48: 1-91. Madrid.

ALBA-TERCEDOR, J. \& A. SANCHEZ-ORTEGA, 1988. Un método rápido y simple para evaluar la calidad biológica de las aguas corrientes basado en el de HELLAWELL (1978). Limnetica, 4: 51-56.

ARGANO, R. 1979. Isopodi (Crustacea Isopoda). Guide per il riconoscimiento delle specie animali delle acque interne italiane. C.N.R. AQ/1/43. Verona.

BASAGUREN, A. 1988. Tricópteros como indicadores de la calidad de las aguas de Bizkaia. Actas Congr. de Biol. Ambiental (II Congr. Mundial Vasco), II: 111-118.

BASAGUREN, A. \& E. ORIVE, 1990. The relationship between water quality and caddisfly assemblage structure in fast-running rivers. The River Cadagua bassin. Environm. Mon. and Assess., 15: 35-48.

BIRSTEIN, J.A. 1964. Fauna of U.S.S.R. Crustacea. Vol. VII, No. 5. Freshwater Isosods (Asellota). Israel Program for Scientific Translations. Jerusalem.

GARCIA DE JALON, D. 1986. Los Hydropsychidae (Trichoptera) de la Cuenca del Duero. Bol. Asoc. ess. Entom., 10: 127-138.

GONZALEZ DEL TANAGO, M. \& D. GARCIA DE JALON, 1984. Desarrollo de un índice biológico para estimar la calidad de las aguas de la Cuenca del Duero. Limnetica, 1: 263-272.

G. DE BIKUÑ̃, B. 1989. Análisis de las características fisico-químicas y de las taxocenosis de Amphipoda $e$ Isopoda (Clase Crustacea) de los ríos de Vizcaya: Tipificación abiótica de la red fluvial en interrelación entre el medio abiótico y biótico. Tesis Doctoral. Universidad de León. (Inédita).

G. DE BIKUÑA, B. \& R. ASENSIO, 1991. Factores que definen el hábitat del isópodo Proasellus meridianus
Racovitza en ríos calcáreos de Bizkaia (Norte de España). Bol. R. Soc. EsU. Hist. Nat. (Sec. Biol.), 87(1-4): 209-221.

HELLAWELL, J. 1986. Biological indicators of freshwater Dollution and enviromental management. Elsevier Applied Science Publishers. London, New York.

HENRY, J.P. \& G. MAGNIEZ, 1978. O. Isopoda. In: Limnofauna europaea (J. ILLIES, ed.) 238-243. G. Fisher Verlag, Stuttgart-N.Y./Swets \& Amsterdam.

HENRY, J.P. \& G. MAGNIEZ, 1983. Crusracés isopodes (principalement Asellotes). Asso. Française de Limnologie. Paris. (In G. DE BIKUÑA \& ASENSIO, 1991).

HYNES, H.B.N. 1960. The biology of polluted waters. Liverpool University Press.

JEFFRIES, M. \& D. MILLS, 1990. Freshwater ecology. Principles and applications. Belhaven Press. London, New York.

LUDWIG, J.A. \& J.F. REYNOLDS, 1988. Statistical ecology. A Urimer on methods and computing. John Wiley \& Sons, New York.

MALTBY, L. 1991. Pollution as a probe of life-history adaptation in Asellus aquaticus (Isopoda). Oikos, 61: 11- 18. MARGALEF, R. 1983. Limnología. Omega. Barcelona.

MASON, C.F. 1984. Biología de la contaminación del agua dulce. Alhambra. Madrid.

PUIG, M.A., J. BAUTISTA, M.J. TORT, \& N. PRAT, 1981 . Les larves de Trichopteres de la riviere Llobregat (Catalogne, Espagne). Distribution longitudinale et relation avec la qualité de I'eau. In: Proc. 3rd. Int. Symp. on Trichoptera (G.P. MORETTI, ed.), Series Entomologica, 20: 303-309.

S.A.S.-UNIV. GRANADA 1991. Los vertidos de aguas residuales urbanas en Andalucía. 21 ed. Foycar. Sevilla.

UNIV. GRANADA 1990. Caracterización físico-químicabiológica de las aguas del Alto Genil. Estudio integral de la calidad y contaminación de las aguas. Dirección General de Obras Hidráulicas del M.O.P.U. (Inédito).

ZAMORA-MUÑOZ, C. 1992. Macroinvertebrados acuaticos. caracterización y calidad de las aauas de los cauces de la cuenca alta del río Genil. Tesis Doctoral. Universidad de Granada. (Inédita). 
APÉNDICE 1. Localidades exactas donde fueron capturados Asellus aquaticus y Proasellus meridianus en la cuenca del Alto Genil (río, localidad, altitud, coordenadas UTM, fecha. número de ejemplares).

APPENDIX 1. Localities in the Alto Genil basin where Asellus aquaticus and Proasellus meridianus were caught (river, locality, altitude, UTM coordinates, date, number of individuals).

\section{Asellus aquaticus}

Río Genil:

ESTACIÓN 5: Chauchina, 550 m, 30SVG3118, Septiembre 1988, 19; Diciembre 1988, 12; Febrero 1989, 4; Junio $1989,7$.

ESTACIÓN 6: Fuente Vaqueros, 540 m, 30SVG2718, Marzo 1988, 3; Junio 1988, 11; Septiembre 1988, 7; Diciembre 1988, 7; Febrero 1989, 6; Junio 1989, 1; Septiembre 1989. 2.

ESTACIÓN 7: Trasmulas, 520 m, 30SVG2317, Marzo 1988, 3; Junio 1988, 35; Septiembre 198\&, 54; Diciembre 1988, 27; Febrero 1989, 12; Junio 1989, 4; Septiembre 1989, 4; Febrero 1990, 7.

ESTACIÓN 8: Brácana, 520 m, 30SVG1718, Marzo 1988, 9; Junio 1988, 33; Septiembre 1988, 25; Diciembre 1988, 9; Febrero 1989, 1; Junio 1989, 3; Septiembre 1989, 4; Febrero 1990, 1.

ESTACIÓN 9: Villanueva de Mesía, 500 m, 30SVG0919, Marzo 1988, 2; Junio 1988, 1; Septiembre 1988, 54; Febrero 1989, 3; Junio 1989, 2; Septiembre 1989, 4.

ESTACIÓN 10: Huétor-Tájar, 480 m, 30SVG0615, Marzo 1988, 4; Junio 1988, 12; Septiembre 1988, 2; Diciembre 1988, 1; Febrero 1989, 2; Junio 1989, 3; Septiembre 1989, 4.

ESTACIÓN 11: Los Infiernos de Loja, 470 m, 30SVG9914, Junio 1988, 2; Septiembre 1988, 5; Diciembre 1988, 1; Febrero 1989, 16; Junio 1989, 4; Septiembre 1989, 5.

ESTACIÓN 12: Loja, 460 m, 30SVG9615, Marzo 1988, 2; Junio 1988, 32; Septiembre 1988, \&; Diciembre 1988, 5; Febrero 1989, 5; Junio 1989, 11; Septiembre 1989, 17.

ESTACIÓN 13: Aguas arriba del embalse de Iznájar, 440 m, 30SVG9418, Septiembre 1988, 10; Diciembre 1988, 2; Febrero 1989, 5; Junio 1989, 2; Septiembre 1989, 1.

Río Cubillas:

ESTACIÓN 5 Desembocadura, 540 m, 30SVG2718, Marzo 1988, 12; Junio 1988, 3; Septiembre 1988, 7; Diciembre 1988, 3 Febrero 1989, 15; Junio 1989.7; Septiembre 19\&- . 1; Febrero 1990, 4.

Arroyo de Vilano:

ESTACIÓN 1: Huétor-Tájar, 500 m, 30SVG0719, Marzo 1988, 1; Junio 1988 11: Septiemb -e 1988, 8; Diciembre 1988, Febrero 1989, 2; Junio 1989, 1.

Arroyo Noniles:

ESTACIÓN 1: Láchar, 540 m, 30SVG2717, Junio 1988, 1; Febrero 1990, 1

Río Genazal:

ESTACIÓN 1 Desembocadura,480 m, 30SUG9516, Marzo 1988, 1; Septiembre 1988. 1; Febrero 1989.

Río Frío:

ESTACIÓN 3: Descmbocadura, 440 m, 30SUG9417, Septiembre 1988, 4; Diciembre 1988, 6; Febrero 1989, 13; Septiembre 1989, 5.

\section{Proasellus meridianus}

Arroyo Manzanil:

ESTACIÓN 1: Desembocadura,500 m, 30SVG0013, 